\title{
Behavioral and Brain Measures of Executive Attention and School Competence in Late Childhood
}

\author{
Purificacion Checa and M. Rosario Rueda \\ Department of Experimental Psychology, University of Granada, \\ Granada, Spain
}

\begin{abstract}
This study examines the role of executive attention on school competence in early adolescence. Twelve-year-old children $(N=37)$ performed a combined Flanker-Go/No-Go task while their brain activation was registered using electroencephalogram (EEG). Additionally, measures of children regulation, schooling skills, and academic achievement were obtained. We observed that individual differences in executive attention and Effortful Control predict most dimensions of school competence. Also, individual differences in the amplitude of event-related potentials (ERPs) related to interference suppression predict school achievement and some skills important for school. The results are consistent with the role attributed to executive attention in self-regulation.
\end{abstract}

A wide variety of everyday situations call for effortful control of behavior in order to adjust actions to goals. The regulation of cognition and action has been considered an important function of attention (Posner \& Rothbart, 1998). Attentional control or executive attention is required in situations that involve overcoming well-learned or dominant responses, facing novel and/or dangerous situations, detecting and correcting errors and planning actions (Posner \& DiGirolamo, 1998). Within Posner's neurocognitive model, executive attention is related to the function of a neural network involving the anterior cingulate cortex and prefrontal regions, called the executive attention network (Posner \& DiGirolamo, 1998; Posner \& Rothbart, 1998).

A basic measure of executive attention in the laboratory is provided by tasks involving conflict among possible responses. An example of these is the Flanker task. In this task, participants are required to respond to a central target and ignore flanking stimuli. The target may consist of an arrow pointing either right or left and the required response is to indicate the direction of the central arrow by pressing a key. Then, flanking arrows point to either the same (congruent trials) or the opposite (incongruent trials) direction than the central arrow. In the incongruent condition, processing of the flankers suggests a response that conflicts with that of the relevant central stimulus. It is thought that resolving conflict requires the selection of the appropriate response and the suppression of competing and interfering incongruent responses which results in slower reaction time and lower accuracy in incongruent compared to congruent conditions (Eriksen \& Eriksen, 1974).

This work was supported by grants from the Spanish Ministry of Science and Innovation to M. Rosario Rueda (reference: PSI2008-02955) and to P. Checa with a predoctoral grant (reference: AP2005-2711).

Correspondence should be addressed to Purificacion Checa, Psicología Experimental y Fisiología del Comportamiento, Universidad de Granada, Campus de Cartuja s/n, 18071 Granada, Spain. E-mail: checaal@ugr.es 
A different task commonly used to study inhibitory control is the so-called Go/No-Go task. It involves presentation of a series of stimuli that require a fast response ("Go" trials), usually pressing a key. Frequent "Go" trials are intermixed with infrequent "No-Go" trials to which participants must hold their response. The frequency of "Go" trials creates a tendency to respond that must be inhibited in No-Go trials, thereby providing a measure of the ability to inhibit a response tendency. However, although Flanker and Go/No-Go tasks involve executive attention they target different processes. For instance, optimal performance of Flanker task requires suppression of interfering information (IS) and optimal performance of Go/No-Go task requires inhibition of preponderant responses (RI) (Bunge, Dudukovic, Thomason,Vaidya, \& Gabrieli, 2002). IS is involved in preventing irrelevant information from being processed and may act at the perceptual or response selection level, whereas RI is a more peripheral process related to response selection and execution (Nigg, 2000).

Previous studies have shown that approximately above age 7 children perform the flanker task at accuracy levels comparable to that of adults (Rueda et al., 2004a; Ladouceur, Dahl, \& Carter, 2007). Despite being able to perform the task without difficulty, children show important individual differences in their reaction time and accuracy levels of performance. Individual differences in the extent to which children are affected by incongruent stimulation in the flanker task have been related to aspects of children' behavior at school, including socialization and achievement (Checa, Rodríguez-Bailón, \& Rueda, 2008).

Contributions of attentional control to self-regulation and schooling have also been examined in the temperament literature. Effortful Control (EC) is a temperamental factor that captures individual differences in the ability to regulate behavior in relation to current and future needs, and reflect individuals' efforts to control impulses according to instructions, goals, or social norms (Rothbart \& Derryberry, 1981). It has been argued that the executive attention network in Posner's model constitutes the neural system underlying EC (Rueda, Posner, \& Rothbart, 2005a). This connection is supported by evidence showing that measures of executive attention through conflict tasks often correlate with parents and/or self-reported measures of EC (Checa et al., 2008; Ellis, Rothbart, \& Posner, 2004; Gerardi-Caulton, 2000; Gonzalez, Fuentes, Carranza, \& Estevez, 2001). In the school context, different studies have shown that individual differences in EC are positively related to academic outcomes (Devinsky, Morrell, \& Vogt, 1995; Ellis et al., 2004), especially particular characteristics such as distractibility, persistence and activity control (Martin, 1994). Also, recent data suggest that EC appears to be a key mediator of the relationship between children's social adjustment to school and academic achievement (Checa et al., 2008).

Several previous studies have used event-related potentials (ERPs) with Go/No-Go and Flanker tasks in adults (Eimer, 1993; Koop, Rist, \& Mattler, 1996) and children (Davis, Bruce, Snyder, \& Carter, 2003; Rueda et al., 2004b) to examine the neural bases of executive attention. It has been found that the $\mathrm{N} 2$ component, a negative deflection registered at frontal channels around 200 to $300 \mathrm{msec}$ post-target, is larger for trials that involve interference suppression (incongruent) and response inhibition (No-Go trials) than for Congruent and Go trials, respectively. In young children, it has been shown that the N2 component in Flanker and Go/No-Go tasks appears to be somewhat delayed compared to adults. The amplitude of the effect is also larger for children than adults. Moreover, the congruency effect is sustained longer in children (Davis et al., 2003; Rueda et al., 2004b). These data seems to indicate that brain mechanisms underlying executive attention become more efficient with age, at least in terms of speed of processing and conflict resolution (Rueda et al., 2004b). 
Brain imaging studies have shown that IS and RI processes are supported by a network of cortical areas involving the dorsal division of the anterior cingulate cortex (dACC) and regions of the dorsolateral prefrontal cortex (DLPFC), ventro lateral prefrontal cortex (VLPFC), and parietal cortex (Corbetta \& Shulman, 2002; Fan, McCandliss, Fossella, Flombaum, \& Posner, 2005). The anatomical network underlying IS and RI appears to overlap to some extent, although some regions are particularly implicated in IS while other regions are involved in RI. A study using functional magnetic resonance imaging (fMRI) showed that RI involves greater recruitment of DLPFC, VLPFC and parietal structures compared to IS, while IS is associated with greater dACC recruitment relative to RI (Blasi et al., 2006). Other studies have found that the VLPFC is implicated in both IS and IR, while additional posterior structures such as the anterior and posterior cingulate cortex and superior and inferior parietal lobe are involved in RI (Bunge et al., 2002).

In the present study we aimed at examining whether brain activation related to IS and RI is associated with children school competence. Different studies have shown that the effortful and voluntary control of behavior is particularly important for school success. Students' attentional control is particularly important in order to regulate behavior and focus on what is being discussed in the classroom despite the presence of potential distracting events. For instance, Passolunghi, Cornoldi, and De Liberto (1999) showed that children exhibiting poorer performance when solving arithmetic tasks have greater difficulty inhibiting irrelevant information than better performers. In consonance with this, other studies have shown that measures tapping executive attention, such as Stroop-like interference and performance on inhibitory control tasks, also show a consistent relationship with arithmetic competence (Blair \& Razza, 2007; Bull, Espy, \& Wiebe, 2008; Bull \& Scerif, 2001). Executive attention appears to have a beneficial effect on learning of school subjects such as literacy and numeracy through its involvement on cognitive flexibility and regulation (Blair \& Razza, 2007; Bull \& Scerif, 2001; Checa et al., 2008; Fuchs et al., 2005; Russell \& Ginsburg, 1984). Although most of these studies have considered attention as a key factor to school success, any of them have examined separately the contribution of interference suppression (IS) and response inhibition (IR) to school competence, neither they have directly assessed the relationship between schooling competence and brain mechanisms underlying those processes.

While a considerable amount of data suggest that systems of self-regulation such as temperamental effortful control and executive attention are important for school competence, to our knowledge, only one study has examined the relationship between brain measures tapping the functioning of the executive attention network and school competence. Hirsh and Inzlicht (2010) have recently shown that the amplitude of the Error-related Negativity (ERN), a post-response ERP component associated with action-monitoring, is related to academic achievement in undergraduate students. They have interpreted this result as indicative of the importance of efficiently engaging cognitive control processes, such as error-detection, when needed in order to monitor learning processes important for academic success.

In the present study, the aim was to measure attentional control using both temperament questionnaires and cognitive and brain measures related to conflict processing and examine its relationship with various aspects of schooling competence. We have used a combined Flanker and Go/No-Go task in order to examine the possible separate contribution of IS and RI to school achievement.

Several prior studies that examined the relationship between executive function and school competence have been conducted with preschoolers (Blair \& Razza, 2007; Bull et al., 2008; 
Bull \& Scerif, 2001; Duncan et al., 2007; Valiente, Lemery-Chalfant, Swanson, \& Reiser, 2008). In our study, we extend the assessment of this relationship to late childhood because there is evidence showing that systems of self-regulation are important for school competence and socialization at this age (Checa et al., 2008).

Temperament measures of EC and cognitive measures of executive attention both target individuals' ability to control behavior, therefore we expected both measures to be related to school competence. Although we view IS and IR as part of the executive attention network, their somewhat different neuroanatomies suggest that the two processes might contribute differently to school competence. Based on previous results (Bull \& Scerif, 2001; Passolunghi et al., 1999) we expected the ability to suppress irrelevant information to be particularly important for achievement in school subjects with high load of reasoning such as mathematics.

Finally, we intended to use the amplitude and latency effects on the ERPs related to interference suppression and response inhibition as indexes of efficiency of underlying brain mechanisms of executive attention. We expected to find an association between the ERPs effects, particularly those related to interference suppression, and measures of school competence.

\section{METHOD}

\section{Participants}

A total of 37 children (mean age: 12.6 years, SD: 0.60; 19 girls) and their parents participated in the study. All participants came from Granada's urban area in South Spain and had a similar social background. Participants were recruited at the school by sending a letter to their caregivers with information about the general purpose of the study. The caregivers of all participants gave written consent to participate in the study. All participants were Caucasian-Europeans except one girl who was African and one girl and one boy who were Hispanic.

\section{Procedure}

The study involved two sessions. In the first session, which was conducted at the school and took approximately 50 minutes, participants filled-up a questionnaire about their schooling skills. Also, the parent-report version of the Early Adolescence Temperament Questionnaire-Revised (EATQ-R; Ellis \& Rothbart, 2001) was given to caregivers with instructions for completing it and returning it to the school with their children in a sealed envelope. In the second session, children were tested individually. At arrival, participants were informed of the general procedure of the session and were given a few minutes to get comfortable with the lab setting before starting. Putting the net on and checking impedances took about 10 minutes. Once wearing the net, children were verbally informed of the instructions to complete the experimental task (described below). Completing the task took about 15-20 minutes. Once the task was completed and the net was taken off, participants completed the Kaufman Brief Intelligence Test (K-BIT; Kaufman \& Kaufman, 2000), which took about 15 minutes. A T-shirt of the lab and other small presents were offered to the children at the end of the experimental session in appreciation for their collaboration. Finally, the board of the school in which children were enrolled provided information on grades obtained at the end of the academic year by the students involved in the study. 


\section{Measures}

Experimental task. We used a combination of the Flanker and Go/No-Go tasks in order to measure processes of interference suppression (IS) and response inhibition (RI) (see Figure 1). Each trial started with a fixation point of variable duration randomly selected between 600 and $1,200 \mathrm{msec}$. Subsequently, a target was presented until a response was made or as long as 2,000 msec. The target display consisted of an arrow pointing either right or left that was flanked by two arrows on each side. For half of the trials the flanking arrows pointed to the same (congruent) direction as the central arrow, and they pointed to the opposite (incongruent) direction for the other half of the trials. The row of arrows could either appear above or below the fixation cross (50\% of the trials on each location). Participants were instructed to indicate the direction of the central arrow by pressing a button in a response pad (the left button for leftward pointing central arrows and right button for central arrows pointing right) as fast as possible only when the arrows were presented in black color (Go condition, $75 \%$ of the trials) and withhold their response when the arrows were presented in red color (No-Go condition, 25\% of the trials). Finally, an errorfeedback tone was provided only when a response was made in No-Go trials. Each participant performed a total of 192 trials divided in three blocks, with a brief break between blocks. The trial condition (whether it was congruent or incongruent and Go or No-Go) was randomly assigned in each trial.

Measure of temperamental effortful control. The Spanish parent-report version of the Early Adolescence Temperament Questionnaire-Revised (EATQ-R; Ellis and Rothbart, 2001) was used to measure children's effortful control. The EC measure in our study was calculated as the average score of the following scales: (1) Activation Control, which reflects the capacity to perform an action when there is strong tendency to avoid; (2) Attention, which reflects the capacity to focus as well as to shift attention when desired; and (3) Inhibitory Control, which reflects the capacity to plan, and to suppress inappropriate response (Ellis \& Rothbart, 2001).

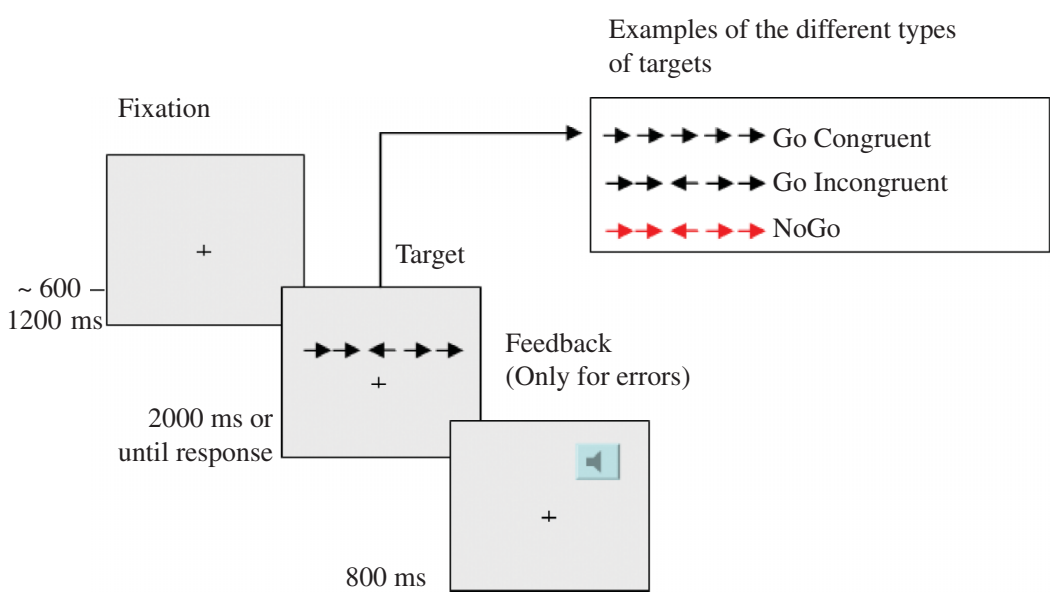

FIGURE 1 Schematic representation of the experimental task used in the study. (color figure available online) 
Items are rated on a 5 -point scale ranging from $1=$ hardly ever true to $5=$ almost always true. The EATQ-R has been reported to have a good reliability. In our sample the internal reliability (measured by Cronbach's alpha) of the EC factor was $\alpha=.80$. Three parents did not return the temperament questionnaire.

Measure of schooling skills. Information on schooling skills was obtained through the self-report version of the Health Resources Inventory (HRI; Juvonen \& Keogh, 1992). This questionnaire consists of 31 items that group onto four factors: Rule following (RF, Cronbach's $\alpha=.79$ in our sample), Student-role Understanding ( $\mathrm{SrU}, \alpha=.80$ ), Sociability ( $\mathrm{SO}, \alpha=.50$ ), and Tolerance to Frustration (TF, $\alpha=.55$ ). The RF factor reflects the student's ability to function within the constraints of the school environment. Sample items for this factor are "I behave well at school" and "I am considerate and polite with others." The SrU factor describes behaviors associated with effective learning such as students' understanding of their duties and responsibilities. Samples items are: "I am interested in the work we do at school" and "I try to complete home-work assignments." The SO factor consists of items reflecting effective interpersonal functioning (e.g., "I have many friends" or "My classmates are fond of me"). Finally, the TF factor measures the individual ability to cope with failure and other social pressures. Sample items are "I can accept things not going my way" and "I face my mistakes." Items are responded in a 5-point Likert scale ranging from never to always each particular item is true of oneself.

Measure of academic achievement. The High School board provided information of the official grades of all grades obtained at the end of the academic year by each student. The average of all grades was calculated as the mean of grades of Life Science, Social Science, Physical Education, Literacy (Spanish), Second language (English), Mathematics, Music, and Technology. Grades in Mathematics and Literacy (Spanish language and grammar) were also examined separately given their central role on school learning and achievement.

Measure of intelligence. We used the Kaufman Brief Intelligence Test (K-BIT; Kaufman and Kaufman, 2000) to measure intelligence. The K-BIT is an individually administered test that consists of two subscales, vocabulary and matrices. The vocabulary scale is a measure of language and experience-related knowledge, and the matrices scale assesses abstract reasoning or fluid intelligence skills. The test provides scores for the two subscales as well as a composite IQ score.

\section{EEG Data Acquisition and ERPs Processing}

Electroencephalogram (EEG) was recorded using the 128-channel Geodesic Sensor Net (www.egi.com). The EEG signal was digitized at $250 \mathrm{~Hz}$. Impedances for each channel were measured prior to recording and kept below $80 \mathrm{k} \Omega$ before testing. Recording was vertex referenced and the time constant value was $0.01 \mathrm{~Hz}$. Data from four children were not included in the analysis due to technical problems during task completion.

To build target-locked ERPs, individual files were filtered using a $0.3-12 \mathrm{~Hz}$ band-pass. Subsequently, continuous data were segmented into 1.2 seconds-long epochs (from $200 \mathrm{msec}$ pre-target to $1,000 \mathrm{msec}$ post-target). Segmented files were scanned for artifacts with the Net Station artifact detection tool using a threshold of 80 micro-volts for eye blinks and eye movement's detection. Segments containing eye blinks or movements as well as segments with more 
than 25 channels with noisy signal (containing abrupt amplitude changes) were discarded for further processing. Data were also visually inspected to ensure the parameters of the artifact detection tool were appropriate for each child. In addition, we used a criterion of a minimum of 15 clean segments per condition among the correctly responded trials for processing individual data. Six children were excluded because they did not reach this criterion. Thus, data from a total of 27 children were used for ERPs analyses.

\section{RESULTS}

We conducted a $t$-test to examine the main effect of Flanker Type and Go/No-Go condition in both behavioral and electrophysiological (amplitude and latency) data. We also calculated correlations between EC (measured by temperamental factor of EC), IS and IR (measured by reaction time, percentage of error and electrophysiological indexes), and school competence. Finally, we conducted regression analyses in order to test the specific contribution of those dependent measures that showed significant correlations with academic outcomes and schooling skills.

\section{Behavioral Results}

Mean (across subjects) of median (per subject) reaction times and percentage of errors were obtained for congruent and incongruent conditions. The difference in reaction time between congruent $(574 \mathrm{msec})$ and incongruent $(648 \mathrm{msec})$ trials was significant $(t(64)=-3.5 ; p<.001)$. A similar result was obtained for percentage of errors, with less percentage of errors in congruent trials $(1.4 \%)$ compared to incongruent trials $(4.3 \%)(t(64)=-.2 .5 ; p<.05)$. Also, the percentage of errors for the Go condition (omission errors) was $0.98 \%$, and $0.84 \%$ for the No-Go condition (commission errors).

\section{ERPs Results}

ERPs for the four experimental conditions included in the task are presented in Figure 2. As expected, we observed a larger negativity for incongruent compared to congruent trials (IS contrast) as well as for No-Go compared to Go trials (RI contrast) in frontal leads. However, the differences appeared at different time-windows. To examine interference suppression (IS) we computed mean amplitudes within a time-window of 460 to $760 \mathrm{msec}$ post target for incongruent and congruent trials. To examine response inhibition (RI) we computed mean amplitudes in a time-window of 330 to $630 \mathrm{msec}$ post-target for Go and No-Go trials. In order to test whether these amplitude differences were larger at midline or lateral sites, mean amplitude of several channels on the left-frontal (F3), midline (Fz), and right-frontal (F4) sites was calculated. The latency was computed as the time elapsing between presentation of the target and the maximum negative peak within the 330-630 msec post-target window for response inhibition, and between presentation of the target and the maximum negative peak within the $460-760 \mathrm{msec}$ post-target for interference suppression.

Separate 2 (Flanker Type: congruent vs. incongruent) $\times 3$ (Electrode Position: left, midline and right) ANOVAs with mean amplitude and latency as dependent measures were conducted. 
a) ERPS

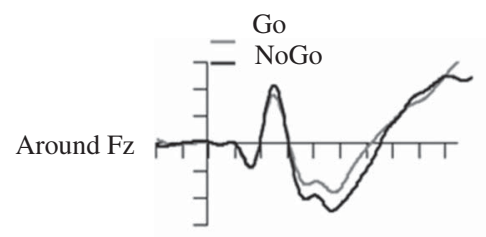

Around $\mathrm{Fz}$

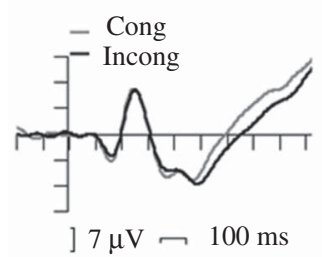

b) Topographic maps

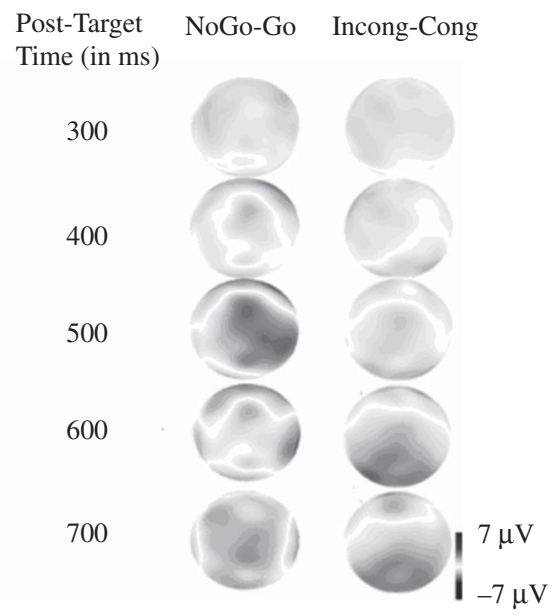

FIGURE 2 (a) Event-related potentials (ERPs). Response Inhibition effect: Go vs. No-Go condition (top of the figure) and Interference Suppression effect: congruent (Congr) vs. incongruent (Incong) trials (bottom of the figure). (b) Topographic maps. Scalp distributions of the difference between Go and No-Go trials (left panel) and the difference between Congruent and Incongruent trials (right panel) at various time points after presentation of the target. Darker color represents areas where larger differences were observed between conditions (either Go vs. No-Go or Congruent vs. Incongruent).

For amplitude, the main effect of Flanker Type was significant $(F(1,26)=13.68 ; p<.001)$, indicating larger negative amplitude for incongruent $(-.8 \mu \mathrm{V})$ compared to congruent $(.23 \mu \mathrm{V})$ trials. The main effect of Electrode Position was also significant $(F(2,52)=3.9 ; p<.05)$ with larger negative amplitude for midline leads compared to right, $(F(1,26)=9.5 ; p<.001)$, or left, $(F(1,26)=3.3 ; p=.08)$, sites. The Flanker Type $\times$ Electrode Position interaction was not significant $(F>1)$. Also, no significant effects were obtained using latency as dependent measure (all $F>1$ ).

Additionally, separate 2 (Trial Condition: Go vs. No-Go) $\times 3$ (Electrode Position: left, midline, and right) ANOVAs were conducted with mean amplitude and latency as dependent measures. Using amplitude, the main effect of Trial Condition was significant, $(F(1,26)=5.6$; $p<.05)$, showing larger negative amplitudes for No-Go $(-3.4 \mu \mathrm{V})$ compared to Go $(-2 \mu \mathrm{V})$ trials. The main effect of Electrode Position was also significant, $(F(2,52)=6.5 ; p<.01)$, with larger negative amplitude for midline, compared to left, $(F(1,26)=9.4 ; p<.01)$ and right, $(F(1,26)=11.9 ; p<.01)$, sites. The Condition $\times$ Electrode Position interaction was not significant. Finally, using peak latency as dependent variable, only the main effect of Electrode Position was significant, $(F(1,52)=3.7 ; p<.05)$, with longer latency of the peak in midline channels compared to leads located on the right, $(F(1,26)=5.4 ; p<.05)$, or left sides, $(F(1,26)=7.5$; $p<.05)$. The Condition $\times$ Electrode Position interaction was not significant $(F>1)$. 


\section{Correlations}

Two scores were obtained as measures of individual efficiency for processes of interference suppression (IS) and response inhibition (RI) for each participant. The interference suppression score was calculated by subtracting the median RT (or \% of errors) for congruent trials from the median RT (or \% of errors) for incongruent trials. The response inhibition score (RI) was computed as the percentage of errors in No-Go trials. Pearson's correlations between these scores and the rest of measures taken in the study were then calculated. The EC was positively related to average of all grades and grades in mathematics and literacy $(r=.56 ; p<.01 ; r=.38 ; p<.05$ and $r=.52 ; p<.01$, respectively). Moreover, EC showed a positive correlation with the school skills of (1) Student-role Understanding ( $r=.44 ; p<.01)$; (2) Sociability $(r=.40 ; p<.05)$; and (3) the average of all schooling skills $(r=.42 ; p<.05)$. We also found that the RT IS score $\left(\mathrm{IS}_{\mathrm{RT}}\right)$ was negatively related to the average of school grades and grades in mathematics and literacy $(r=-.37 ; p<.05, r=-.39 ; p<.01$ and $r=-.36 ; p<.05$, respectively). Moreover, a positive correlation between the RI score $\left(\mathrm{RI}_{\mathrm{ER}}\right)$ and the IS score calculated with the percentage of errors $\left(\mathrm{IS}_{\mathrm{ER}}\right)$ was found $(r=.35 ; p<.05)$. Finally, scores of the intelligence subscales of Vocabulary and Matrices and IQ composite were negatively related to $\mathrm{IS}_{\mathrm{RT}}$ score $(r=-.35$; $p<.05 ; r=-.55 ; p<.01$ and $r=-.50 ; p<.01$, respectively) and positively related to Effortful control (EC) $(r=.35 ; p<.05 ; r=.33 ; p<.05$ and $r=.38 ; p<.01$; respectively). Also, the average of school grades and grades in mathematics and literacy were positively related to subscale of Vocabulary $(r=.53 ; p<.01, r=.61 ; p<.001$ and $r=.51 ; p<.01$, respectively), subscale of Matrices $(r=.67 ; p<.001$ and $r=.68 ; p<.001$ and $r=.58 ; p<.01$, respectively) and IQ composite $(r=.66 ; p<.001$ and $r=.71 ; p<.001$ and $r=.60 ; p<.001$, respectively).

We also calculated electrophysiological effects of interference suppression (ERP-IS) and response inhibition (ERP-RI) for each participant. The ERP-IS score was calculated by subtracting the mean amplitude for congruent trials from the mean amplitude for incongruent trials within the 460 to $760 \mathrm{msec}$ time window. The ERP-RI score was calculated by subtracting the mean amplitude for Go trials from the mean amplitude for No-Go trials in a time window of 330 to $630 \mathrm{msec}$ post-target. The ERP-IS score was negatively correlated with grades in mathematics $(r=-.42 ; p<.05)$, as well as Rule Following $(r=-.39 ; p<.05)$, Student-role Understanding scores $(r=-.47 ; p<.05)$, and the average of all schooling skills $(r=-.39 ; p<.05)$. No significant correlations were obtained between the ERP-RI score and any other measure taken in the study.

\section{Regressions}

To examine the unique contribution of the dependent measures that showed significant correlations with academic outcomes and schooling skills we conducted stepwise multiple regression analysis. We conducted two sets of regression analyses separately for measures of school achievement (average of grades and grades in literacy and math) and the various measures of schooling skills as dependent variables. For the first sets of analysis, (see Table 1), scores of intelligence subscales of vocabulary (VO) and matrices (MA) were entered first, followed by Effortful Control (EC) scores, then the ISRT score and the electrophysiological measure of IS (ERP-IS). 
TABLE 1

Regression Predicting Academic Achievement

\begin{tabular}{|c|c|c|c|c|c|c|c|}
\hline & \multicolumn{3}{|c|}{ No Including IQ } & \multirow[b]{2}{*}{ Dependent Variable } & \multicolumn{3}{|c|}{ Including $I Q$} \\
\hline & \multicolumn{3}{|c|}{$\Delta R^{2}$ of the Model .37} & & \multicolumn{3}{|c|}{$\Delta R^{2}$ of the Model .57} \\
\hline & $\beta$ & $F$ & $p$ & Average of All Grades & $\beta$ & $F$ & $p$ \\
\hline VO & & & & & .28 & 1.89 & .07 \\
\hline MA & & & & & .56 & 4.5 & .001 \\
\hline EC & .51 & 3.6 & .001 & & .38 & 3.12 & .01 \\
\hline \multirow[t]{3}{*}{ ERP-IS } & -.30 & -2.1 & .05 & & -.02 & -.11 & .91 \\
\hline & \multicolumn{3}{|c|}{$\Delta R^{2}$ of the model .16} & & \multicolumn{3}{|c|}{$\Delta R^{2}$ of the model .55} \\
\hline & $\beta$ & $F$ & $p$ & Math & $\beta$ & $F$ & $P$ \\
\hline VO & & & & & .14 & .75 & .46 \\
\hline MA & & & & & .64 & 4.4 & .001 \\
\hline EC & .20 & 1.01 & .32 & & -.07 & -.47 & .64 \\
\hline IS RT & -.31 & -1.6 & .11 & & .14 & .74 & .47 \\
\hline ERP-IS & -.44 & -2.3 & .05 & & -.35 & -2.4 & .05 \\
\hline
\end{tabular}

VO: vocabulary; MA: Matrices; EC: Effortful Control; IS: Interference suppression; ERP-IS = mean of peak amplitude for ERP from incongruent minus mean of peak amplitude for event-related potentials from congruent trials. Significant effects $(p<.05)$ are marked in boldface.

For the second set of analysis the order of variables was the same except for not including the VO and MA scores (see Table 2).

Results showed that EC and ISRT were significant predictors of the average of all grades and the EC remained significant after controlling by intelligence (see Table 1). Also, the brain measure of interference suppression (ERP-IS) was a significant predictor of grades in mathematics even after controlling by intelligence (see Table 1). Only EC was a significant predictor of grades in literacy $(\beta=.49 ; p<.01)$, which remained significant after controlling by intelligence $(\beta=.36 ; p<.05)$.

In addition, we found that EC and marginally the ERP-IS score were significant predictors of the skill of Student-role Understanding and the average of all schooling skills (see Table 2) in both sets of analyses, controlling and not controlling by measures of intelligence. Finally, only the ERP-IS accounted for unique variance when predicting Rule Following.

\section{DISCUSSION}

In the present study, we measured the ability to exercise control over one's behavior from the temperamental perspective, using a parent-reported questionnaire, and from the neurocognitive perspective by means of behavioral and electrophysiological indexes of performance of a combined Flanker and Go/No-Go task. We have observed that individual differences in temperamental Effortful Control (EC) show a similar pattern of correlations with intelligence, schooling 
TABLE 2

Regression Predicting Schooling Skills

\begin{tabular}{|c|c|c|c|c|c|c|c|}
\hline & \multicolumn{3}{|c|}{ No Including IQ } & \multirow[b]{2}{*}{ Dependent Variable } & \multicolumn{3}{|c|}{ Including IQ } \\
\hline & \multicolumn{3}{|c|}{$\Delta R^{2}$ of the Model .17} & & \multicolumn{3}{|c|}{$\Delta R^{2}$ of the Model .24} \\
\hline & $\beta$ & $F$ & $p$ & Average of School Skill & $\beta$ & $F$ & $p$ \\
\hline VO & & & & & .06 & .30 & .76 \\
\hline MA & & & & & -.29 & -1.5 & .15 \\
\hline EC & .45 & 2.4 & .05 & & .38 & 2.1 & .05 \\
\hline \multirow[t]{3}{*}{ ERP-IS } & -.33 & -1.8 & .09 & & -.33 & -1.8 & .08 \\
\hline & \multicolumn{3}{|c|}{$\Delta R^{2}$ of the model .36} & & \multicolumn{3}{|c|}{$\Delta R^{2}$ of the model .36} \\
\hline & $\beta$ & $F$ & $p$ & Student-role understanding & $\beta$ & $F$ & $p$ \\
\hline VO & & & & & .04 & .21 & .83 \\
\hline MA & & & & & -.01 & -.03 & .97 \\
\hline EC & .40 & 2.3 & .05 & & .40 & 2.3 & .05 \\
\hline \multirow[t]{3}{*}{ ERP-IS } & -.43 & -2.5 & .05 & & -.43 & -2.5 & .05 \\
\hline & & & & & \multicolumn{3}{|c|}{$\Delta R^{2}$ of the model .36} \\
\hline & & & & Rule following & $\beta$ & $F$ & $p$ \\
\hline VO & & & & & .34 & 1.9 & .07 \\
\hline MA & & & & & .14 & .76 & .45 \\
\hline ERPs-IS & -.39 & -2.1 & .05 & & -.39 & -2.1 & .05 \\
\hline
\end{tabular}

VO: vocabulary; MA: Matrices; EC: Effortful Control; IS: Interference suppression; ERP-IS = mean of peak amplitude for ERP from incongruent minus mean of peak amplitude for event-related potentials from congruent trials. Significant effects $(p<.05)$ are marked in boldface.

skills and academic achievement as measures of IS. This was expected given the fact that EC and behavioral measures of IS are usually correlated (Checa et al., 2008; Ellis et al., 2004; Rothbart \& Rueda, 2005). Numerous empirical data (Checa et al., 2008; Ellis et al., 2004; Gerardi-Caulton, 2000; Gonzalez et al., 2001) have supported the relationship between temperamental EC and the functional efficiency of the executive attention network mostly measured through performance of tasks involving IS. This type of evidence has been previously used to argue that the executive attention network is the neural circuit supporting EC (Rothbart \& Rueda, 2005). Several temperamental studies have pointed to the importance of aspects such as persistence, distractibility and activity level to success in school (Martin, 1994; Keogh, 1983). There is also evidence showing that the influence of EC on school competence is not the same at different ages (Maziade, Coté, Boutin, Boudreault, \& Thivierge, 1986). Keogh has pointed out that certain temperament characteristics that conform EC, such as perseverance and control over distracting information, gains on importance when the material to be learned is more complex and the school tasks are larger and more difficult (Keogh, 2003). For instance, Guerin and colleagues (1994) showed that high levels of perseverance and low levels of distractibility are related to school success in early adolescence. Our behavioral results are in consonance with this data because we also 
found a negative relationship between the degree of interference from distracting stimulation in the flanker task and school grades.

We also found a positive correlation between reaction time indexes of IS and IR. This was expected given that both processes are related to the executive attention network. However, only the ability to suppress irrelevant information (IS) showed a clear relationship with school competence. The behavioral measure of IS (IS $\mathrm{RT}_{\mathrm{RT}}$ ) is correlated with intelligence scores in both the vocabulary and matrices subscales. Likewise, $\mathrm{IS}_{\mathrm{RT}}$ shows a significant correlation with achievement in school subjects. Both of these correlations indicate that larger interference scores, and thus poorer ability to suppress distracting information, are related to poorer achievement at school and lower intelligence scores. Nonetheless, we did not obtain significant correlations between the RI measure and neither intelligence nor any of the variables related to school competence. The pattern of results is similar when RI and IS are assessed at the electrophysiological level. The ERP-IS index shows a significant correlation with grades in math and some schooling skills, whereas the ERP-RI index does not correlate with any of the schooling variables taken into account in our study. These data show an interesting dissociation between two types of inhibitory processes and measures relevant for school success. Our data suggest that the ability to inhibit processing of irrelevant information, but not the more peripheral form of inhibition necessary to stop a motor response, is important for aspects of schooling that involve both fluid and crystallized intelligence, learning of school material and displaying skills that promote school success. However, prior studies have shown that performance of inhibitory tasks is related to a variety of aspects of schooling competence (Blair \& Razza, 2007; Bull \& Scerif, 2001; Fuchs et al., 2005; Russell \& Ginsburg, 1984). In our view, RI processes may be more important for stopping motor responses and controlling impulses that are necessary for schooling skills such as sitting still or taking turns to talk, and perhaps not so central to skills involving reasoning and cognitive flexibility. Further research is needed in order to clarify the relation between processes of RI and school competence.

Regression analyses conducted with our dataset show that EC and IS, the last one measured at both behavioral and electrophysiological level, are significant predictors of grades at school (see Table 1). On the one hand, temperamental EC is a significant predictor of the average of school grades and literacy independently of whether intelligence was controlled for or not. We consider that this is because EC is a broader measurement of self-regulatory abilities than IS. EC scores capture individual differences in a larger range of behaviors including patterns of responses that children display at home and other social settings and that are observed and reported by their parents. On the other hand, the effect of $\mathrm{IS}_{\mathrm{RT}}$ is not significant after controlling for fluid intelligence (Matrices subscale) when predicting the average of all grades. We believe that fluid intelligence and IS share a considerable amount of variance provided that tasks requiring interference suppression and those involving fluid intelligence have been shown to activate quite overlapping neural structures (Crone, Wendelken, van Leijenhorst, Honomichl, Christoff, \& Bunge, 2009; Duncan et al., 2000). Besides, we consider that attentional control is particularly important when it comes to solving tasks with a high load of reasoning. In this respect, our data also show that the ERP index for interference suppression is a significant predictor of grades in mathematics but not in literacy. Solving mathematic problems requires representing information in working memory, shifting attention between elements relevant for solving the problem, and inhibiting elements within the problem that are irrelevant to the purpose of finding the correct solution as well as other potentially distracting stimulation. These processes might be less relevant for literacy in 
forth grade which mainly involves learning of school material, such as literary style, grammar, and vocabulary. Previous studies carried out with preschoolers had shown that the inhibition of irrelevant information is an important predictor of literacy (Blair \& Razza, 2007; Duncan et al., 2007). These two results might appear incongruent; however, during the preschool years literacy is mostly related to pre-reading and reading skills which might well rely upon processes such as representing and monitoring information in working memory. Phonological processes as well as letter knowledge and word recognition may require to a greater extend the inhibition of irrelevant information (McClelland et al., 2007) in order, for example, to choose the correct sound that match a particular letter or combination of them.

Interestingly, our data show that the ERP-IS amplitude effect is a significant predictor of grades in math even after controlling for fluid intelligence (see Table 1). This is also true when it comes to predicting schooling skills related to following rules and understanding school duties (Table 2). This result complies evidence of a direct connection between two separate levels of analysis related with attentional control and, more broadly, with self-regulation. On the one hand, the assessment of neural mechanisms through the recording of electrical activity coming from cortical neurons while a task requiring attentional control is being performed. On the other hand, the measurement of individual differences in particular behavioral aspects important for adjustment and accomplishment at the school as reported by teachers (grades) or self-reported (schooling skills). Prior developmental studies using the flanker task with ERPs have shown that larger ERP-IS effects are related to immature frontal function (Rueda et al., 2004b). Compared to adults, children appear to show larger amplitude differences between congruent and incongruent conditions in the flanker task. Besides, the effect is sustained longer for children than adults. This has been interpreted as children's greater engagement of frontal structures in order to solve conflict produced by incongruent flankers. Data from our study indicate that children with more adult-like patterns of brain activation during performance of an interference suppression task are the ones showing greater accomplishment at school.

\section{CONCLUSIONS AND IMPLICATIONS FOR EDUCATION}

Results of the present study show a link between efficiency of the executive attention network as evaluated with RT and electrophysiological data and school competence. Particularly, individual differences in the efficiency with which students suppress the interference created by irrelevant information appear to be an important skill for school success. Electrophysiological data indicate that the students who show more mature patterns of brain activation during suppression of irrelevant information appear to use more appropriate strategies for dealing with school demands. Data obtained in this study provide evidence about the importance of executive attention for education. This type of evidence has the potential to guide the design of intervention programs aiming at enhancing school readiness. Our data point out the importance of promoting executive attention and effortful control in order to help children to be successful at school.

In the past years, there have been some efforts to improve children's attention abilities through training. It has been shown that training of attention and other forms of executive functions, such as working memory produces beneficial effects during development (Rueda, Rothbart, McCandliss, Saccomanno, \& Posner, 2005b; Klingberg, et al., 2005). Compared to untrained controls, children who underwent training of attention improved reasoning abilities and showed 
a more adult-like pattern of activation of the executive attention network (Rueda et al., 2005b). Although this type of research has provided encouraging evidence on the effectiveness of interventions, future research will be needed in order to test whether attention training results in better academic performance and schooling competence.

\section{REFERENCES}

Blair, C., \& Razza, R. P. (2007). Relating effortful control, executive function, and false belief understanding to emerging math and literacy ability in kindergarten. Child Development, 78, 647-663.

Blasi, G., Goldberg, T. E., Weickert, T., Das, S., Kohn, P., Zoltick, B., . . Mattay, V. S. (2006). Brain regions underlying response inhibition and interference monitoring and suppression. European Journal of Neuroscience, 23, 1658-1664.

Bull, R., Espy, K. A., \& Wiebe, S. A. (2008). Short-term memory, working memory, and executive functioning in preschoolers: Longitudinal predictors of mathematical achievement at age 7 years. Developmental Neuropsychology, $33(3), 205-228$.

Bull, R., \& Scerif, G. (2001). Executive functioning as a predictor of children's mathematics ability: Inhibition, switching, and working memory. Developmental Neuropsychology, 19, 273-293.

Bunge, S. A., Dudukovic, N. M., Thomason, M. E., Vaidya, C. J., \& Gabrieli, J. D. E. (2002). Immature frontal lobe contributions to cognitive control in children: Evidence from fMRI. Neuron, 33, 1-20.

Checa, P., Rodríguez-Bailón, R., \& Rueda, M. R. (2008). Neurocognitive and temperamental systems of self-regulation and early adolescents' school competence. Mind, Brain and Education, 2, 177-187.

Corbetta, M., \& Shulman, G. L. (2002). Control of goal-directed and stimulus-driven attention in the brain. Nature Reviews Neuroscience, 3, 201-215.

Crone, E. A., Wendelken, C., van Leijenhorst, L., Honomichl, R. D., Christoff, K., \& Bunge, S. A. (2009). Neurocognitive development of relational reasoning. Developmental Science, 12, 55-66.

Davis, E. P., Bruce, J., Snyder, K., \& Carter, N. (2003). The X-trials: Neural correlates of an inhibitory control task in children and adults. Journal of Cognitive Neuroscience, 15, 432-443.

Devinsky, O., Morrell, M. J., \& Vogt, B. A. (1995). Contributions of anterior cingulate to behaviour. Brain and Cognition, 118, 279-306.

Duncan, G. J., Dowsett, C. J., Claessens, A., Magnuson, K., Huston, A. C., Klebanov, P., et al. (2007). School readiness and later achievement. Developmental Psychology, 43, 1428-1446.

Duncan, J., Seitz, R. J., Kolodny, J., Bor, D., Herzog, H., Ahmed, A., et al. (2000). A neural basis for general intelligence. Science, 289, 457-460.

Eimer, M. (1993). Effects of attention and stimulus probability on ERPs in a Go/No-go task. Biological Psychology, 35, $123-138$.

Ellis, L. K., \& Rothbart, M. K. (2001). Revision of the Early Adolescent Temperament Questionnaire. Poster presented at the 2001 Biennal Meeting of the Society for Research in Child Development, Minneapolis, Minnesota.

Ellis, L. K., Rothbart, M. K., \& Posner, M. I. (2004). Individual differences in executive attention predict self-regulation and adolescent psychosocial behaviors. New York, NY: New York Academy of Sciences.

Eriksen, B. A., \& Eriksen, C. W. (1974). Effects of noise letters upon the identification of a target letter in a nonsearch task. Perception \& Psychophysics, 16, 143-149.

Fan, J., McCandliss, B. D., Fossella, J., Flombaum, J. I., \& Posner, M. I. (2005). The activation of attentional networks. Neuroimage, 26, 471-479.

Fuchs, L. S., Compton, D. L., Fuchs, D., Paulsen, K., Bryant, J. D., \& Hamlett, C. L. (2005). The prevention, identification, and cognitive determinants of math difficulty. Journal of Educational Psychology, 97, 493-513.

Gerardi-Caulton, G. (2000). Sensitivity to spatial conflict and the development of self-regulation in children 24-36 months of age. Developmental Science, 3, 397-404.

Gonzalez, C., Fuentes, L. J., Carranza, J. A., \& Estevez, A. F. (2001). Temperament and attention in the self-regulation of 7-year-old children. Personality \& Individual Differences, 30, 931-946.

Guerin, D., Gottfried, A., Oliver, P., \& Thomas, C. (1994). Temperament and school functioning during early adolescence. The Journal of Early Adolescence, 14, 200-225.

Hirsh, J. B., \& Inzlicht, M. (2010). Error-related negativity predicts academic performance. Psychophysiology, 47, 192-196. 
Juvonen, J., \& Keogh, B. K. (1992). Children's and teacher's views of school-based competence and their relation to children's peer status. School Psychology Review, 21, 410-423.

Kaufman, A. S., \& Kaufman, N. L. (2000). K-BIT, Test breve de inteligencia de Kaufman. Madrid, Spain: TEA.

Keogh, B. (2003). Temperament in the classroom: Understanding individual differences. Baltimore, MD: P.H. Brookes.

Klingberg, T., Fernell, E., Olesen, P. J., Johnson, M., Gustafsson, P., Dahlstrom, K., et al. (2005). Computerized training of working memory in children with ADHD-A randomized, controlled trial. Journal of the American Academy of Child \& Adolescent Psychiatry, 44, 177-186.

Koop, B., Rist, F., \& Mattler, U. (1996). N200 in the flanker task as a neurobehavioral tool for investigating executive control. Psychophysiology, 33, 282-294.

Ladouceur, C. D., Dahl, R. E., \& Carter, C. S. (2007). Development of action monitoring through adolescence into adulthood: ERP and source localization. Developmental Science, 10, 874-891.

Martin, R. P. (1994). Children temperament and common problems in schooling: Hypotheses about causal connection. Journal of School Psychology, 32, 119-134.

Maziade, M., Coté, R., Boutin, P., Boudreault, M., \& Thivierge, J. (1986). The effect of temperament on longitudinal academic achievement in primary school. Journal of the American Academy of Child Psychiatry, 25(5), $692-696$.

McClelland, M. M., Cameron, C. E., Connor, C. M., Farris, C. L., Jewkes, A. M., \& Morrison, F. J. (2007). Links between behavioral regulation and preschoolers' literacy, vocabulary, and math skills. Developmental Psychology, 43, 947-959.

Nigg, J. T. (2000). On inhibition/disinhibition in developmental psychopathology: Views from cognitive and personality psychology and a working inhibition taxonomy. Psychological Bulletin, 126, 220-246.

Passolunghi, M. C., Cornoldi, C., \& De Liberto, S. (1999). Working memory and intrusions of irrelevant information in a group of specific poor problem solvers. Memory and Cognition, 27, 779-790.

Posner, M. I., \& DiGirolamo, G. J. (1998). Executive attention: Conflict, target detection, and cognitive control. Cambridge, MA: The MIT Press.

Posner, M. I., \& Rothbart, M. K. (1998). Attention, self-regulation and consciousness. Philosophical Transactions of the Royal Society of London, 353, 1915-1927.

Rothbart, M. K., \& Derryberry, D. (1981). Development of individual differences in temperament. In M. E. Lamb \& A. L. Brown (Eds.), Advances in developmental psychology (Vol. 1, pp. 37-86). Hillsdale, NY: Erlbaum.

Rothbart, M. K., \& Rueda, M. R. (2005). The development of effortful control. In U. Mayr, E. Awh, \& S. W. Keele (Eds.), Developing individuality in the human brain. A tribute to Michael I. Posner (pp. 167-188). Washington, DC: American Psychological Association.

Rueda, M., Fan, J., McCandliss, B. D., Halparin, J. D., Gruber, D. B., Lercari, L. P., et al. (2004a). Development of attentional networks in childhood. Neuropsychologia, 42(8), 1029-1040.

Rueda, M., Posner, M. I., \& Rothbart, M. K. (2005a). The development of executive attention: Contributions to the emergence of self-regulation. Developmental Neuropsychology, 28, 573-594.

Rueda, M. R., Posner, M. I., Rothbart, M. K., \& Davis-Stober, C. P. (2004b). Development of the time course for processing conflict: An event-related potentials study with 4 year olds and adults. BMC Neuroscience, 5, 39.

Rueda, M. R., Rothbart, M. K., McCandliss, B. D., Saccomanno, L., \& Posner, M. I. (2005b). Training, maturation, and genetic influences on the development of executive attention. Proceedings of the National Academy of Sciences of the USA, 102, 14931-14936.

Russell, R. L., \& Ginsburg, H. P. (1984). Cognitive analysis of children's mathematical difficulties. Cognition and Instruction, 1, 217-244.

Valiente, C., Lemery-Chalfant, K., Swanson, J., \& Reiser, M. (2008). Prediction of children's academic competence from their effortful control, relationships, and classroom participation. Journal of Educational Psychology, 100, 67-77. 